\title{
VISUALIZAÇÃO DA VENTILAÇÃO NATURAL EM ENSAIOS NA MESA D'ÁGUA COMPARADO A SIMULAÇÕES COMPUTACIONAIS
}

\section{Visualization of natural ventilation in water table test compared to computational simulations}

\author{
Ana Clara de Almeida Xavier ${ }^{1}$ \\ Universidade Estadual de Maringá, Maringá, PR, Brasil, anaclaraxavier48@gmail.com \\ Izabella Hafele Gularte ${ }^{2}$ \\ Universidade Federal de Santa Catarina, Florianópolis, PR, Brasil, izabella.gularte@gmail.com \\ Martin Ordenes Mizgier ${ }^{3}$ \\ Universidade Federal de Santa Catarina, Florianópolis, PR, Brasil, martin.ordenes@ufsc.br \\ Marieli Azoia Lukiantchuki ${ }^{4}$ \\ Universidade Estadual de Maringá, Maringá, PR, Brasil, malukiantchuki2@uem.br
}

\begin{abstract}
Resumo
No processo de projeto nota-se a existência de uma lacuna entre a teoria e a prática, principalmente com relação à ventilação natural. Isso requer o uso de métodos que auxilie a visualização do fluxo de ar nos edifícios e sua transferência para os projetos. Muitas das ferramentas confiáveis de predição da ventilação natural, como o túnel de vento e os softwares de Dinâmica dos Fluídos Computacional (Computational Fluid Dynamic - CFD), são complexas e caras, dificultando o seu uso durante o ensino e na prática dos projetistas. Diante disso, o objetivo dessa pesquisa é verificar se ferramentas simplificadas representam o escoamento do ar no projeto arquitetônico de maneira fiel. Para isso, a visualização da ventilação natural através de experimentações simplificadas é comparada com ferramentas complexas e de alta confiabilidade, como simulações CFD. O método foi dividido em 3 etapas. Primeiramente, foi construído um modelo físico modular, baseado na literatura especializada. Em seguida, ensaios na mesa d'água foram executados. Por fim, simulações computacionais em uma ferramenta simplificada (software Fluxovento) e em uma mais complexa baseada na Dinâmica dos Fluídos Computacional (software CFX) foram realizadas. Os resultados demonstram a compatibilidade entre as simulações CFD e os ensaios na mesa d'água, indicando que o ensino com essa ferramenta torna acessível o entendimento de conceitos básicos da ventilação natural. Já no Fluxovento, diferenças significativas foram registradas na análise do fluxo de ar, dificultando o entendimento da ventilação natural. Ressalta-se a característica modular do modelo para elaborar novos casos em estudo de ventilação natural.
\end{abstract}

Palavras-chave: Ventilação natural. Projeto arquitetônico. Mesa d’água. Simulações computacionais.

\begin{abstract}
During the design process, there is a gap between theory and practice, especially concerning natural ventilation. This gap requires simplified methods to aid the airflow visualization in buildings and its implementation over the design process. Many of the reliable natural ventilation tools, such as wind tunnel and CFD software, are complex and expensive, making it difficult to use for the designers. This research aims to verify if simplified and easily accessible tools represent in a trustworthy way the airflow in the architectural project. For this, the visualization of natural ventilation concepts through simplified experimentation is compared with complex and highly reliable tools such as CFD simulations. The methodology of this research was established in 3 stages. First, a modular physical model was built based on the specialized literature. Experimental tests on an intuitive and qualitative tool, such as the water table, were performed. Finally, computational simulations were performed in a simplified tool (Fluxovento) and Computational Fluid Dynamics (CFD) software. The results show compatibility between CFD simulations and water table tests, showing that using this tool for teaching basic concepts of natural ventilation is adequate In Fluxovento software. Significant differences were registered in the qualitative analysis of airflow. We highlight the possibility of taking advantage of the model's modular characteristic to elaborate on new cases in natural ventilation study.
\end{abstract}

Keywords: Natural ventilation. Architectural design. Water table tests. Computational simulations.

How to cite this article:

XAVIER, A. C. DE A.; GULARTE, I. H.; MIZGIER, M. O.; LUKIANTCHUKI, M. A. Visualização da ventilação natural em ensaios na mesa d'água comparado a simulações computacionais. PARC Pesquisa em Arquitetura e Construção, v. 11, p. e020020, 30 set. 2020. DOI: https://doi.org/10.20396/parc.v11i0.8656954. 


\section{Introdução}

A ventilação natural consiste no deslocamento de ar através do edifício, pela disposição de aberturas em suas fachadas, originado por diferenças de pressão entre as áreas externas e internas, podendo ocorrer pela ação dos ventos, pela diferença de temperatura entre o interior e o exterior (efeito chaminé) ou pela combinação de ambos. A ventilação natural em regiões de clima quente e úmido, como a maior parte do território brasileiro, é uma estratégia eficiente para obtenção do conforto térmico por meios passivos, além de contribuir para a redução do consumo de energia elétrica (BITTENCOURT; CÂNDIDO, 2008; LAMBERTS; DUTRA; PEREIRA, 2014).

O incremento da ventilação nos ambientes dependerá, entre outros fatores, da tipologia arquitetônica adotada e das características das aberturas. Para Koenigsberger et al. (1974), Kukreja (1978) e Olgyay (1963), a configuração do fluxo de ar no interior da edificação será determinada por três fatores principais: 1. o tamanho e a localização das aberturas; 2. o tipo e a configuração das aberturas, e 3. a localização de elementos próximos às aberturas, tais como protetores solares e divisórias internas. Sobre o último item, Bittencourt e Cândido (2008) pontuam que a localização inadequada desses elementos pode afetar negativamente na velocidade e na distribuição interna do ar. Para Gratia, Bruyere e Herde (2004), Garrilho da Graça e Linden (2016) e Perén et al. (2014), além da posição, tamanho e tipo das aberturas, o direcionamento e a velocidade dos ventos externos determinam a ventilação natural em um ambiente.

Lawson (2005) explica o ato de projetar como uma tentativa de solucionar previamente problemas de diferentes níveis de dificuldade. Sobre isso, Toledo (1999) destaca a importância do estudo da ventilação natural ocorrer desde o início do projeto e na formação dos alunos. No entanto, é de conhecimento comum as dificuldades existentes em transferir o conhecimento teórico para a prática, principalmente quando se trata do conforto ambiental que muitas vezes não é abordado na concepção do projeto.

Segundo Maciel (2006) e Omrami et al. (2017) apesar das vantagens da integração dos conceitos bioclimáticos aos edifícios, existe uma dificuldade em sua aplicação. Kowaltowski et al. (1998) ressaltam que um dos maiores problemas para a incorporação dos conceitos de conforto é a dificuldade da representação visual desses fenômenos e de sua transposição para os projetos, principalmente com relação à ventilação natural. Omrami et al. (2017) afirmam que prever o comportamento da ventilação natural é um processo complexo devido aos parâmetros físicos envolvidos. Para Trindade, Pedrini e Duarte (2010) as restrições do uso da ventilação natural pelos arquitetos são devido à falta de garantia de que esta estratégia gera conforto, ainda mais se comparada com os sistemas de resfriamento artificial. Sobre isso, Garrilho da Graça e Linden (2016) destacam que os projetistas utilizam resfriamento mecânico por aparentemente serem mais confiáveis para o alcance do conforto térmico dos usuários.

De acordo com Kowaltowski et al. (2006) a visualização dos aspectos de conforto no processo de projeto é um importante instrumento para uma clara transferência desses conhecimentos da teoria para a prática projetual. Para uma conexão entre os aspectos ambientais, oriundos de pesquisa, e as soluções de projeto, é fundamental visualizar os fenômenos por imagens estimulantes ao processo criativo. Para Omrami et al. (2017) a fase mais importante para considerar a ventilação natural é a concepção do projeto, sendo necessário, ainda nesta etapa, o uso de métodos que permitam uma melhor compreensão do fenômeno, tornando o uso da ventilação acessível aos alunos e profissionais por meio de recursos eficazes e de fácil aplicação (ARAÚJO, 2011). A predição do fluxo de ar pode ser realizada principalmente de duas formas: 1. 
experimentos em túnel de vento e em mesas d'água e 2. simulações computacionais com base em dinâmica dos fluidos (CALAUTIT; HUGHES, 2014).

Os programas CFD são pouco acessíveis e de difícil utilização pelos alunos e projetistas, uma vez que exigem um alto investimento inicial e conhecimentos específicos de modelagem computacional. O túnel de vento também é de difícil acesso devido ao elevado custo de execução e dificuldade de operação (MATSUMOTO; LABAKI, 2011; ETHERIDGE; SANDBERG, 1996). Já a mesa d'água é um equipamento mais acessível, possuindo um grande potencial de uso para fins didáticos e práticos. Segundo Toledo e Pereira (2003) e Rossi et al. (2019), as vantagens do equipamento são: a) baixo custo de execução; b) facilidade de operação e manutenção; c) facilidade de uso de maquetes pelos arquitetos e d) visualização continuada do escoamento. No entanto, existem limitações, tais como: a) visualização em apenas duas dimensões, simplificando a avaliação do fenômeno real que é tridimensional; b) impossibilidade de mensuração de parâmetros como a pressão e c) não permitem a análise da ventilação natural por diferença de temperatura. Para Toledo e Pereira (2003) esses equipamentos são pouco explorados para a visualização da ventilação natural, apesar de constatações de possuírem um grande potencial de utilização para fins didáticos e práticos

Outras ferramentas simplificadas da ventilação natural estão surgindo, tais como o software Fluxovento, apresentado em 2005 pelos pesquisadores Carlos Vitor de Alencar Carvalho; Luiz Fernando Martha e Walter Teixeira da PUC-Rio. Esse software foi desenvolvido com base em técnicas de computação gráfica interativa e conceitos de Dinâmica dos Fluidos Computacional. O desenvolvimento numérico do sistema considera um fluxo em regime permanente, não viscoso e incompressível; os efeitos de turbulência e variações de temperatura não foram considerados na análise numérica e a ferramenta permite apenas a análise bidimensional (CARVALHO; MARTHA; TEIXEIRA, 2005). Diante das diversas ferramentas existentes, Fortuna (2000) ressalta que o uso de métodos numéricos não descarta as análises experimentais. Em um projeto que envolva escoamento dos fluidos é comum as técnicas se complementarem, confirmando ou não a compatibilidade das ferramentas.

Desta forma, diante da dificuldade em utilizar ferramentas complexas, de alto custo e que demandam conhecimentos específicos, buscam-se incorporar, no ensino, métodos simplificados que auxiliem os alunos no entendimento dos conceitos básicos da ventilação natural durante a concepção projetual. É possível obter o conhecimento teórico dos fenômenos através das bibliografias existentes, mas poucos princípios reais são testados pelos alunos a respeito da aplicabilidade das soluções nos edifícios. Assim, o objetivo dessa pesquisa é verificar se ferramentas simplificadas representam o fluxo de ar no projeto arquitetônico de maneira fiel. Para isso, a visualização de conceitos básicos de ventilação natural através de experimentações simplificadas é comparada com ferramentas complexas e de alta confiabilidade, como simulações CFD.

\section{Método}

O método adotado nesta pesquisa foi composto de três etapas: 1. definição dos modelos analisados; 2 . ensaios na mesa d'água e 3. simulações computacionais.

\section{Definição dos modelos analisados}

Seis diferentes configurações de aberturas e divisórias internas foram avaliadas, com base em Garcia e Fuentes (2005), a fim de analisar o impacto de seus diferentes tamanhos e posições no desempenho do fluxo de ar interno. Os modelos apresentam dimensões de 6 módulos x 6 módulos, altura de 1 módulo, sendo 1 módulo =1,om. As aberturas e as divisórias foram definidas em função dessa modulação. A escolha por 
modelos simplificados existentes na literatura facilita a comparação entre diferentes ferramentas e o entendimento do fenômeno da ventilação natural. Os modelos foram ensaiados considerando os ventos externos com ângulo de incidência perpendicular aos modelos $\left(0^{\circ}\right)$, conforme a Figura 1.

Figura 1 - Modelos selecionados para as análises

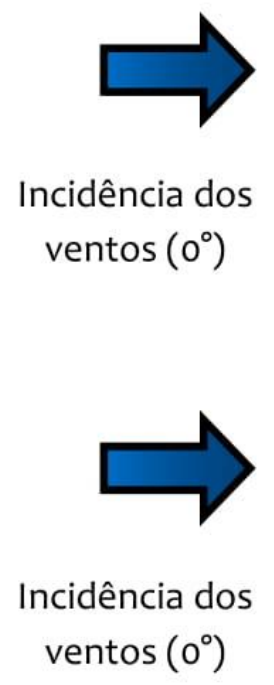

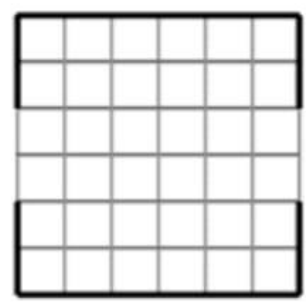

Modelo 01

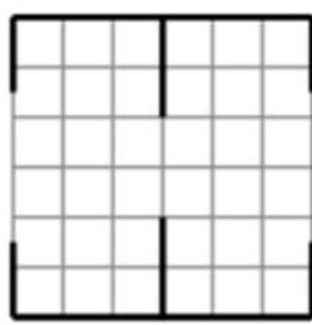

Modelo 04

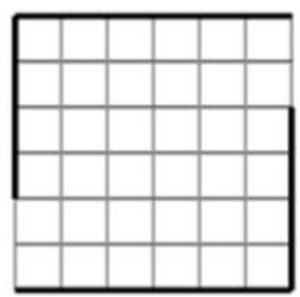

Modelo 02

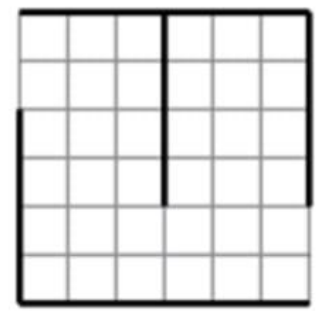

Modelo 05

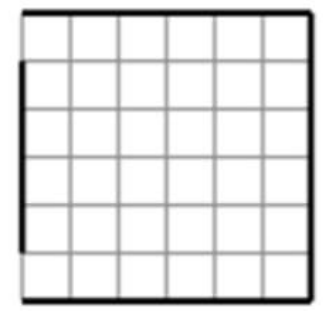

Modelo 03

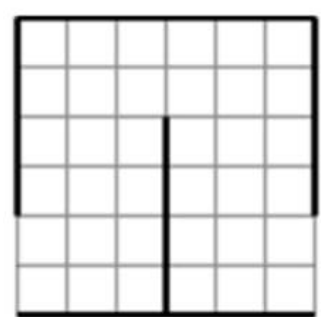

Modelo 06

Fonte: os autores.

\section{Ensaios na mesa d'água}

A mesa d'água permite a visualização do fluxo de ar em modelos reduzidos, utilizando como fluído a água, misturada com um indicador (detergente). Assim, o fluxo de ar é caracterizado de forma qualitativa, apresentando os princípios da ventilação natural nos ambientes internos. Após a definição das configurações, foi elaborado um modelo físico reduzido para a realização dos ensaios. O modelo foi planificado, no software Corel Draw na versão X8 (2016), de forma compatível com a cortadora a laser Automatisa Prisma 6ow, do FABLAB PRONTO 3D, do Departamento de Expressão Gráfica da Universidade Federal de Santa Catarina. Esse processo, além de acelerar essa etapa, facilita a precisão no corte de peças, garantindo uma boa vedação dos encaixes. Como a visualização do fenômeno acontece apenas em duas dimensões, podendo ocorrer tanto em planta quanto em corte, neste caso o modelo foi construído em planta baixa usando o acrílico transparente de $2 \mathrm{~mm}$, que além de ser um material resistente e impermeável, facilita a visualização do fenômeno analisado. A maquete foi construída com encaixes do tipo macho e fêmea, visando maior flexibilidade na modificação das diferentes configurações (Figura 2).

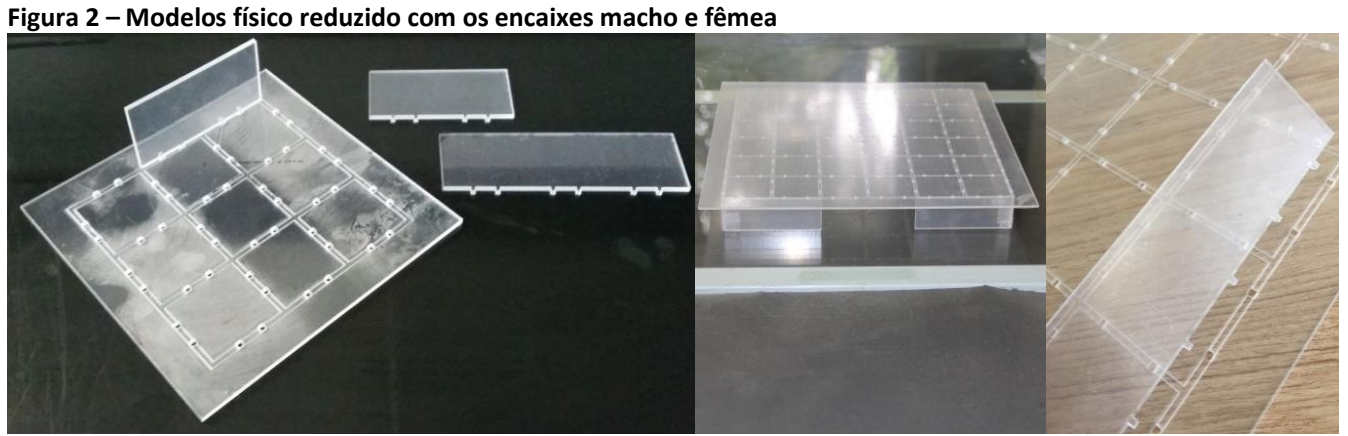


Segundo Toledo e Pereira (2003), a escala das maquetes deve obedecer às limitações da largura da mesa, a fim de impedir a interferência das paredes laterais do canal no escoamento. Utilizou-se a mesa d'água do Laboratório de Conforto Ambiental do Instituto de Arquitetura e Urbanismo da USP, São Carlos (IAU/USP), cuja área de ensaio apresenta $1,10 \mathrm{~m} \times 0,74 \mathrm{~m}$. As dimensões do modelo são de $29 \mathrm{~cm} \times 29 \mathrm{~cm}$ nas bordas exteriores, na escala 1:75, cujas paredes de espessura de $2 \mathrm{~mm}$ equivalem à dimensão de $15 \mathrm{~cm}$ na referida escala. $O$ tamanho do modelo se adequa à largura da mesa, permitindo uma distância de $22,5 \mathrm{~cm}$ das laterais; $25 \mathrm{~cm}$ à barlavento e $57 \mathrm{~cm}$ a sotavento (Figura 3).

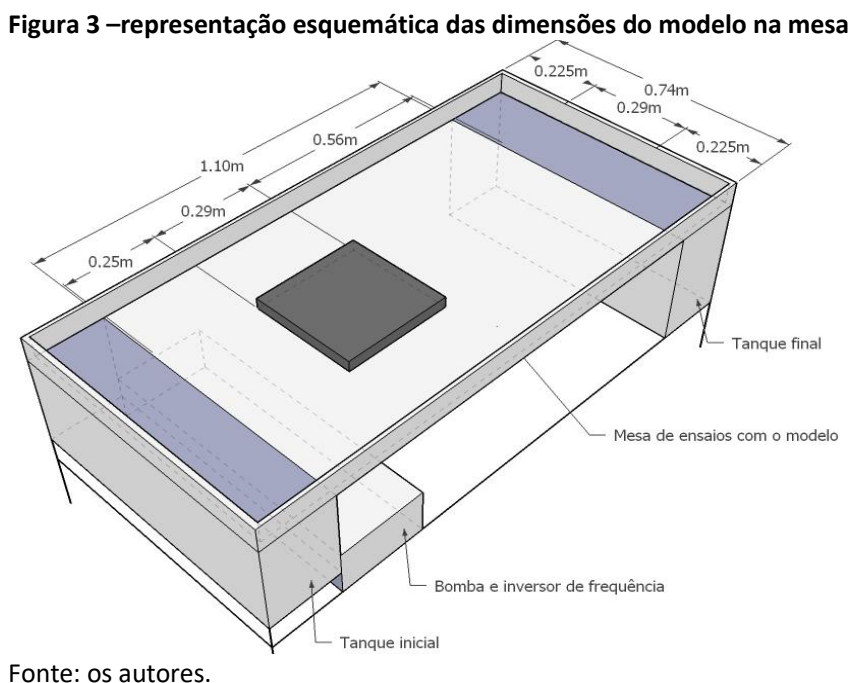

A mesa d'água é composta por dois tanques de água com capacidade de 92,5 litros cada, conectados por uma bomba $0,75 \mathrm{CV}$ e $60 \mathrm{~Hz}$. Um inversor de frequência, modelo CFWo8 da WEG, é responsável por controlar a velocidade e a frequência do fluxo de água. Para a realização dos ensaios, os reservatórios foram cheios e o indicador foi acrescido (Figura 4). A fim de garantir o turbilhonamento necessário para a formação da espuma, a frequência do sistema elétrico foi elevada a $50 \mathrm{~Hz}$ e, após alguns minutos, a frequência foi ajustada para $20 \mathrm{~Hz}$. Esse procedimento, segundo Toledo e Pereira (2003), é adequado para visualizações analógicas de fluxo laminar. Os autores apontam a necessidade do aumento periódico da frequência do inversor e, assim, do turbilhonamento durante os testes, devido à rápida perda de consistência da espuma.
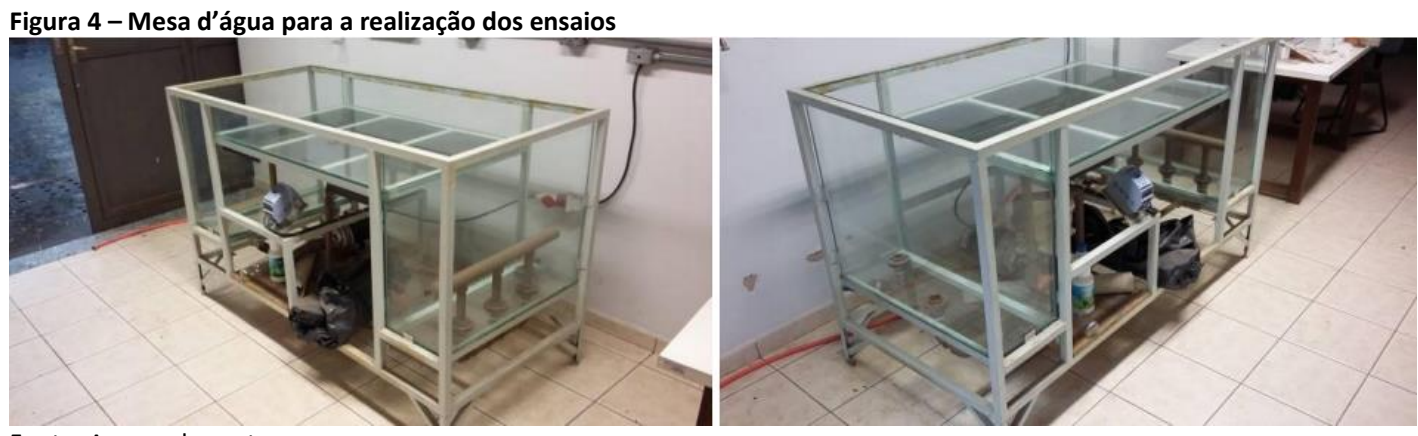

Fonte: Acervo dos autores.

Existem duas diferenças entre as ferramentas utilizadas nesse trabalho: 1. A mesa d'água e o Fluxovento fornecem apenas dados qualitativos, enquanto as simulações CFD possibilitam a aquisição de parâmetros qualitativos e quantitativos. Assim, a comparação entre as ferramentas será unicamente qualitativa; 2 . O fluído utilizado nos experimentos para análise da ventilação natural é a água, enquanto nas simulações 
utiliza-se o ar. Segundo Blessmann (2011) a água e o ar apresentam, sempre que não houver separação da camada limite, um comportamento próximo a um fluido ideal (incompressível e sem viscosidade), apresentando efeitos visuais semelhantes acerca de muitos aspectos. Além disso, o autor enfatiza que se o Re (Equação 1) for constante e as similaridades geométricas e de orientação dos objetos analisados em relação ao escoamento forem asseguradas, a semelhança dinâmica e as forças em pontos correspondentes em dois escoamentos distintos serão, por sua vez, constantes.

$$
R e=\frac{\rho v \mathrm{D}}{\mu}
$$

Onde:

$\rho:$ massa específica do fluído $\left(\mathrm{kg} / \mathrm{m}^{3}\right)$

$\mu$ : viscosidade dinâmica do fluído $\left(\mathrm{m}^{2} / \mathrm{s}\right)$

v: velocidade média do fluído $(\mathrm{m} / \mathrm{s})$

D: área da abertura do modelo $\left(\mathrm{m}^{2}\right)$

Visando igualar o Re dos ensaios, cujo fluído é água, com o das simulações CFD, com o ar, foi realizado um teste relacionando a frequência da mesa com a velocidade do fluxo do fluido. Através dos testes, tem-se a média de valores para a relação frequência $(\mathrm{Hz})$ e velocidade (m/s) (Figura 5). Na mesa d'água utilizou-se a frequência de $35 \mathrm{~Hz}$, correspondendo à uma velocidade de $0,22 \mathrm{~m} / \mathrm{s}$. Em seguida, calculou-se o Re (equação 1), com a incerteza do fluido da mesa não ser água pura. Por fim, visando igualar o valor desse parâmetro na mesa d'água com o das simulações com o ar, adotou-se o mesmo Re, obtendo-se, assim, o valor de velocidade do ar de $0,33 \mathrm{~m} / \mathrm{s}$ como dado de entrada das simulações CFD. O Re é um valor, desde que mantido constante, que permite trabalhar com diferentes fluidos obtendo um comportamento análogo do fluxo em pontos correspondentes. Essa relação não foi estabelecida no Fluxovento, pois este não permite a velocidade do ar como um dado de entrada fornecido pelos usuários.

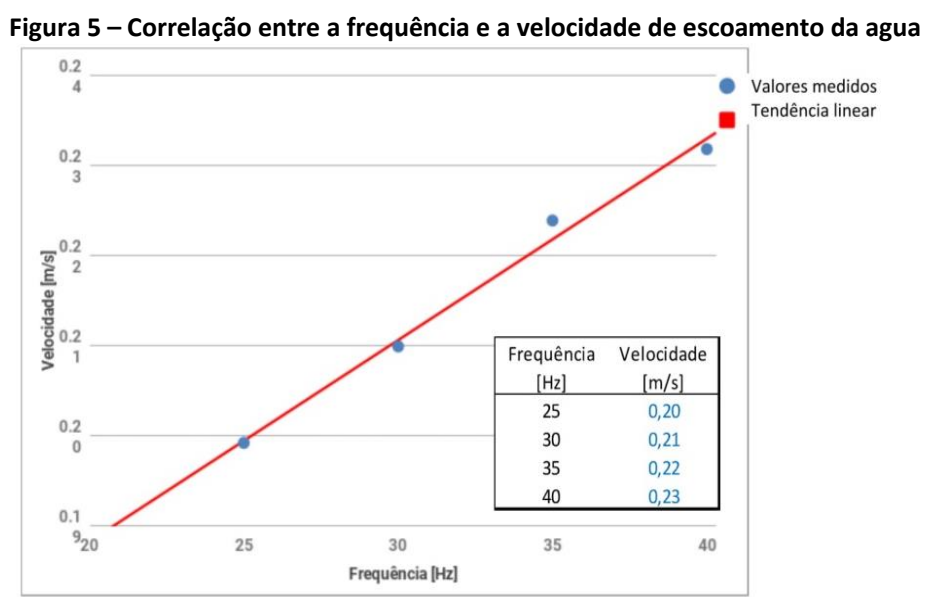

Fonte: os autores.

Tanto a filmagem quanto a captura de imagens foi garantida a partir da instalação de uma câmera digital da marca Nikon, modelo D5100, em um sistema de tripé disposto sobre uma mesa de apoio.

\section{Simulações computacionais}

Para as simulações no software Fluxovento, tem-se um processo simplificado de montagem, sendo os modelos desenhados por linhas que definem as paredes, as divisórias internas e as aberturas. O software apresenta ferramentas de controle de 
edição, para a confecção dos modelos, e controle de visualização, para os resultados. Com o desenho finalizado, executa-se a simulação. As condições de contorno são definidas na forma de vetores de velocidade e o sentido do fluído é determinado pela posição do desenho, seguindo a mesma direção dos ensaios experimentais (Figura 6).

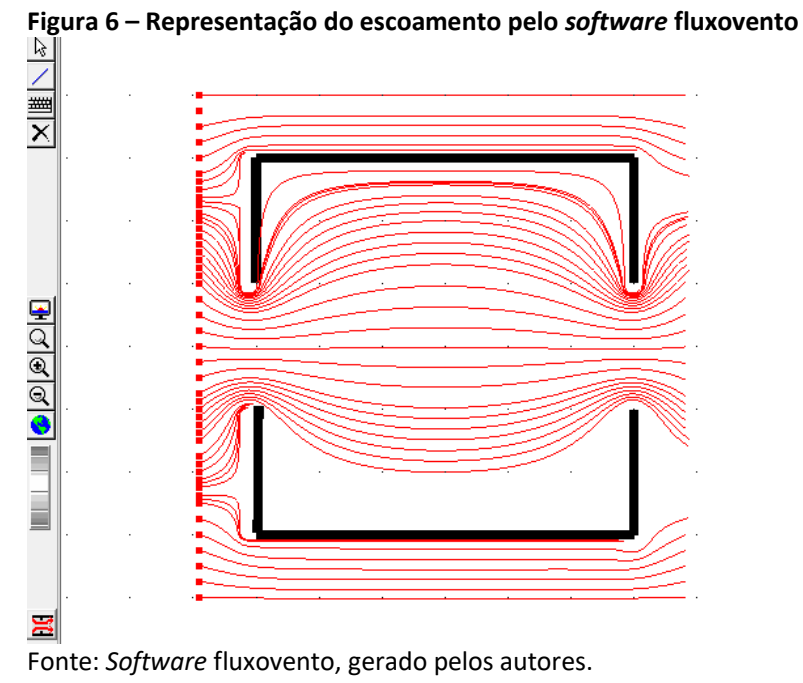

Em seguida, utilizou-se o software de Dinâmica dos Fluidos Computacional (CFD) - CFX (versão 12.0). A confecção dos modelos e do domínio computacional foi realizada no software Autocad (2017), exportando-os, posteriormente, para o Icem-CFX. O domínio, segundo Cóstola (2006), é o volume do espaço para o qual o escoamento será resolvido e foi construído em um formato retangular, cujas dimensões seguiram as recomendações de Harries (2005): (a) distâncias a barlavento e nas laterais $=5 \mathrm{H}(5 \mathrm{~m})$; (b) altura $=6 \mathrm{H}(6 \mathrm{~m}) ;(\mathrm{c})$ distância a sotavento = entre $15 \mathrm{H}(15 \mathrm{~m})$ e $10 \mathrm{H}(10 \mathrm{~m}) ; \mathrm{e}(\mathrm{d})$ sendo $\mathrm{H}=1 \mathrm{~m}$, que corresponde à altura do modelo (Figura 7 ). A área de obstrução do edifício no domínio foi de 1,1\%, atendendo ao recomendado por Franke et al. (2004), que seria menor que $3 \%$, a fim de evitar o efeito de blocagem, impedindo que as fronteiras do domínio influenciem no escoamento.

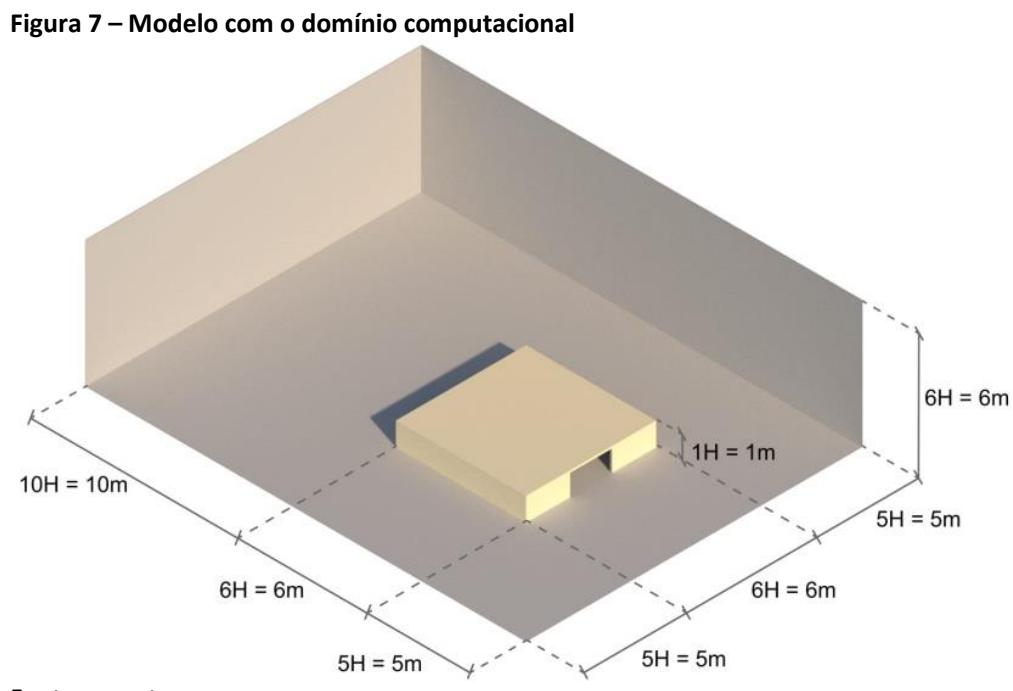

Fonte: os autores.

A precisão dos resultados alcançados na simulação CFD depende da qualidade da malha computacional e tem implicação no nível de convergência (CALAUTIT et al., 2013; HUGHES; CHAUDHRY; CALAUTIT, 2014). Os parâmetros da malha (Figura 8) e as 
condições iniciais e de contorno foram baseados em pesquisas sobre ventilação natural utilizando o CFX (CÓSTOLA, 2006, LEITE, 2008; PRATA, 2005) e são detalhadas no Quadro 1. Por fim, a simulação é calculada no CFX-Solver, e os resultados são visualizados por meio de imagens, gráficos e tabelas no CFX-Post.

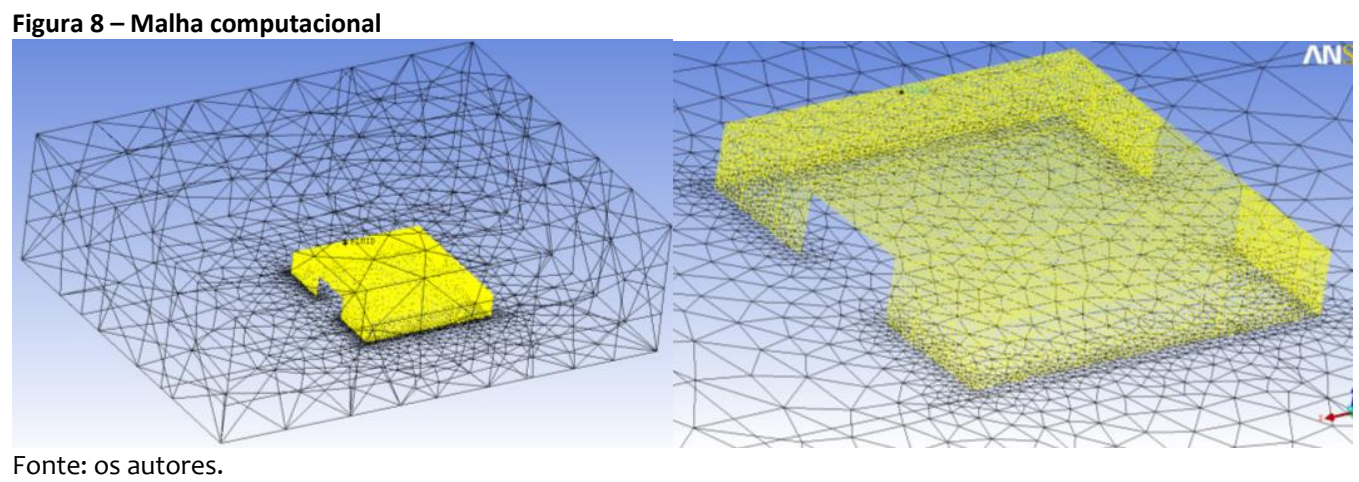

Quadro 1 -Dados de entrada das simulações CFD

\begin{tabular}{|c|l|}
\hline Condição & \multicolumn{1}{|c|}{ Descrição } \\
\hline \multirow{3}{*}{ Malha computacional } & Tipo: estruturada tetraédrica \\
& Parâmetros: maximum element size 16; natural size 4; e cells in gap 8, com refinamento de \\
& $\begin{array}{l}\text { 0,15 m nas superfícies do edifício, a fim de melhorar a visualização do fluxo de ar no espaço } \\
\text { interno e no entorno imediato ao edifício - a combinação desses parâmetros determina a } \\
\text { quantidade de elementos da malha e, assim, o tempo de processamento das simulações. }\end{array}$ \\
\hline \multirow{3}{*}{ Condições do domínio } & $\begin{array}{l}\text { Entrada: INLET, saída: OUTLET; laterais e o teto: WALL free slip (sem atrito), pois não impõe } \\
\text { resistência às partes do domínio onde não são realizadas as análises; e o piso e as superfícies } \\
\text { do edifício: WALL no slip (com atrito). } \\
\text { *as condições do domínio foram definidas de modo semelhante às da mesa d'água }\end{array}$ \\
\hline Regime adotado na simulação & Permanente \\
\hline Modelo de transferência de calor & Isotérmica - $25^{\circ} \mathrm{C}$ \\
\hline Modelo de turbulência & $\begin{array}{l}\text { K-epsilon - comum e bem estabelecido em diversas pesquisas de ventilação natural. } \\
\text { Os parâmetros adotados para a turbulência foram: intensidade média (5\%) - o valor padrão } \\
\text { do software; a high resolution advection scheme e conservative auto timescale. }\end{array}$ \\
\hline Nível de convergência & Maximo de $10^{-4}$ \\
\hline Número de iterações & Valores mínimo e máximo de 600 e 6.000, respectivamente \\
\hline
\end{tabular}

\section{Análise dos resultados}

Para a análise qualitativa da ventilação natural, as imagens geradas pelas três ferramentas analisadas foram comparadas. Os ensaios na mesa d'água foram filmados e, posteriormente as cenas dos vídeos foram convertidas em imagens sequenciais, capturando todos os detalhes dos ensaios. Para o fluxovento, foram utilizadas as imagens de linhas de correntes definidas pelo próprio software, sem a possibilidade de definição de altura de planos de análises. Para as simulações CFD, foi gerado um plano horizontal, localizado no centro das aberturas $(0,50 \mathrm{~m})$. Nesses planos foram plotados os vetores de intensidade e direção do fluxo de ar. Foi utilizada uma escala em que cada cor representa um valor de velocidade correspondente. Os resultados qualitativos foram analisados em cima da malha proposta para a construção dos modelos, visando facilitar o entendimento dos fenômenos de escoamento, tais como: regiões com concentração e rarefação do fluxo de ar; movimentos desuniformes; entre outros. Por fim, desenhos qualitativos simplificando os padrões do ar interno, foram esboçados de forma empírica com flechas, visando uma semelhança com exemplos da literatura.

Posteriormente, para a análise do desempenho quantitativo, inseriram-se pontos em cotas específicas no centro de cada módulo da malha, obtendo-se, por meio de uma equação específica no CFX-Post, os valores da velocidade do ar interno. Em seguida, com 
uma metodologia similar à de Givoni (1976), calculou-se o valor da velocidade do fluxo de ar em pontos do espaço interno e, posteriormente, dividiu-se cada valor pela velocidade do fluxo de ar externo ao modelo, obtendo-se, assim, o aproveitamento (\%) do fluxo de ar interno em relação a velocidade do ar externo.

\section{Resultados}

Primeiramente o tempo de execução de cada etapa foi mapeado, visando uma comparação da aplicabilidade entre as ferramentas. Os experimentos na mesa d'água demandaram um tempo maior para a definição do modelo físico reduzido, tanto na planificação digital como na definição da flexibilidade com os encaixes do tipo macho e fêmea. Após essa etapa, o corte das peças e a montagem do modelo foram facilitados em virtude do maior rigor na etapa anterior. Esse processo durou 2 semanas. Em seguida, a execução dos ensaios de cada modelo, considerando o ajuste da frequência do fluxo de água e da câmera para registro dos ensaios, demorou de 5 a 7 min. Já as simulações CFD demandam um tempo maior. A confecção dos modelos no AutoCAD é rápida e sem dificuldade, por serem geometrias simplificadas. No entanto, a adequação das geometrias, a definição da malha e das condições iniciais e de contorno, são etapas que demandam maior tempo e um maior conhecimento do usuário. Definido isso, a simulação de cada modelo foi executada, demorando em torno de 60 a $90 \mathrm{~min}$. Por fim, o Fluxovento é um processo mais rápido e simplificado. Tanto a definição dos modelos quanto à execução da simulação, para cada caso, demandou um tempo em torno de 5 min.

Nota-se claramente que os experimentos da mesa d'água, embora demandam maior cuidado na realização do modelo físico reduzido, são facilitados pela proximidade que os estudantes têm com maquetes. Além disso, os ensaios não apresentam dificuldade, além de serem rápidos se comparados com as simulações CFD, que demandam um tempo muito maior e um conhecimento aprofundado de Dinâmica dos Fluídos Computacional. Dessa forma, as simulações CFD se tornam menos acessível aos alunos de graduação, comparado com a mesa d'água. Já o Fluxovento é uma ferramenta que além de ser gratuita é rápida e o seu uso é extremamente facilitado, o que em questão de viabilidade se apresenta como uma boa opção ao ensino da graduação.

No entanto, a aplicabilidade não é suficiente para o seu uso no ensino, uma vez que os resultados precisam ser confiáveis na representação do fenômeno da ventilação natural. Em uma análise quantitativa, a figura 9 apresenta os valores da velocidade média do fluxo de ar interno registrados nas simulações CFD. A velocidade média do ar indica que os modelos com ventilação cruzada apresentam desempenho da ventilação interna similar (em torno de $0,09 \mathrm{~m} / \mathrm{s}$ e $0,11 \mathrm{~m} / \mathrm{s}$ ), com pequenas oscilações. Já o modelo com ventilação unilateral (4) apresenta um valor significativamente inferior (0,02m/s).

No entanto, a compreensão da ventilação natural pela velocidade média do fluxo de ar interno não é representativa, pois, tem-se uma diferença na distribuição do fluxo de ar nos diversos pontos internos e, assim, na sensação do conforto térmico dos usuários. Dessa forma, é possível determinar, por exemplo, o layout interno, em função das regiões melhor atingidas pela corrente de ar. Em função disso, visando um maior entendimento pelos alunos dos conceitos da ventilação natural, é importante a possibilidade da visualização do fenômeno por meio de análises qualitativas. Por fim, como forma de complementar essas análises, foi realizada análises quantitativas com a porcentagem de aproveitamento dos ventos externos nos diferentes pontos do ambiente interno, por meio das simulações CFD. 


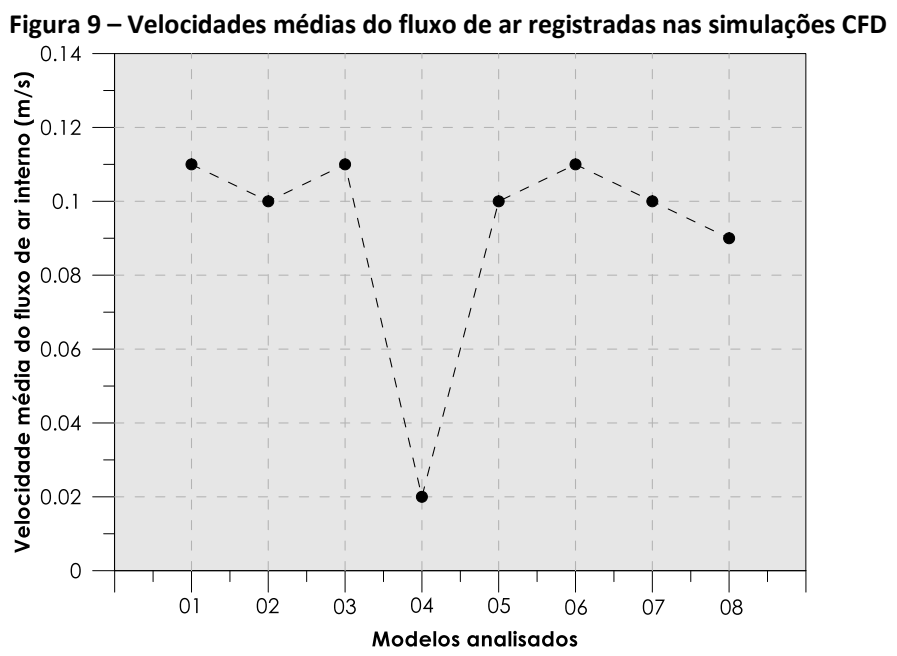

Fonte: os autores.

Qualitativamente, a comparação dos resultados da mesa d'água com as simulações mostra semelhanças e discrepâncias na reprodução do fenômeno físico do escoamento do ar. Para o caso um (Figura 10), nota-se claramente uma distribuição simétrica do fluxo de ar interno, devido à incidência perpendicular do vento na abertura e à configuração das aberturas. Nos dois módulos centrais tem-se um escoamento do ar com altas velocidades, constatando-se o efeito Venturi, com o aumento significativo da velocidade do fluído ( $124 \%$ em relação à velocidade externa) ao passar por um obstáculo de menor dimensão (abertura). Na simulação CFD esse comportamento é visível pelos vetores com maiores velocidades e proximidade, enquanto na mesa d'água isso é notado pela maior concentração de espuma. No entanto, percebe-se uma diferença entre as duas ferramentas na maleabilidade do fluído. Na mesa d'água o escoamento é orgânico, saindo dos dois módulos centrais da malha. Já no CFD, a trajetória é linear, mantendo-se exclusivamente nessa região central. Nesse caso, a mesa d'água e o CFD apresentam divergências decorrentes de uma análise bidimensional e, principalmente, pela diferença do fluído. Nas porções laterais do espaço (dois módulos superior e inferior) há uma redução do fluxo de ar interno (aproveitamento em torno de $8 \%$ e $4 \%$ ), sendo constatado o mesmo nos experimentos devido à ausência de espuma. Esse mesmo comportamento foi constatado em estudos realizados por Olgyay (1963), Givoni (1976), Evans (1980), Gratia, Bruyere e Herde (2004) e Da Graça, Daish e Linden (2015), mostrando uma compatibilidade de ambas ferramentas com a literatura. Já no Fluxovento, nota-se uma diferença significativa na distribuição do ar interno, visto que este atinge todo o ambiente. Além disso, nota-se certa assimetria entre a região superior e inferior, o que não seria compatível com uma configuração de aberturas centrais e em paredes opostas, diferindo das simulações CFD e da mesa d'água. Esse resultado deixa dúvidas sobre a representação do fenômeno com essa ferramenta. 


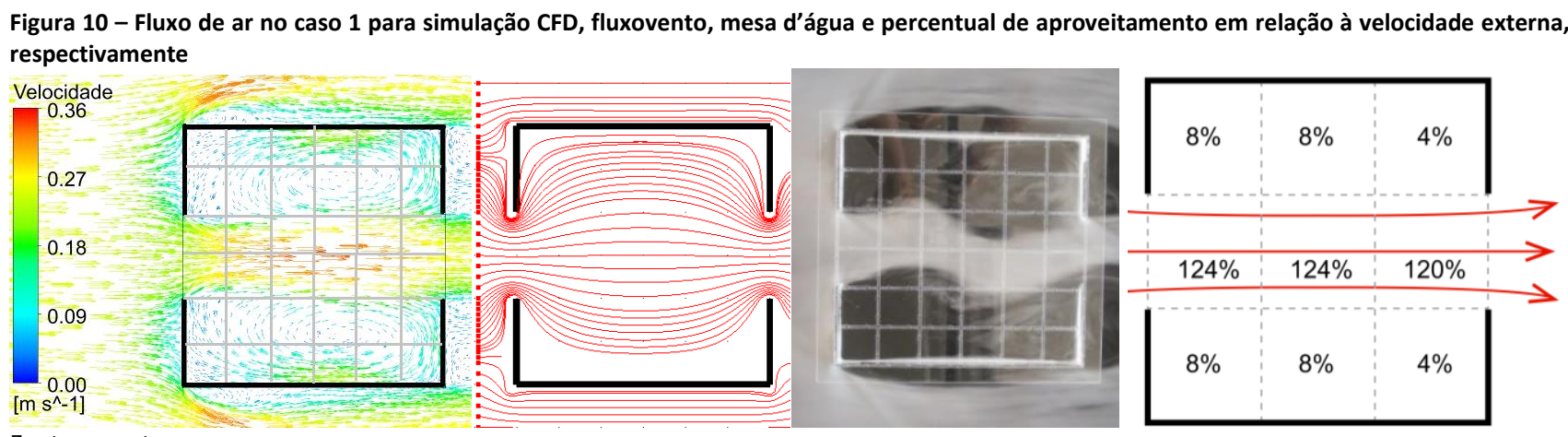

Fonte: os autores.

No caso 2 têm-se um fluxo de ar na diagonal, atingindo principalmente as duas fileiras de baixo e as duas colunas da direita até a abertura de saída (aproveitamento de $80 \%$ ). É nítido que uma região da malha de $1 \mathrm{x} 1$ no canto inferior e a região superior ( 4 módulos x 4 módulos da malha) possui o ar estagnado (aproveitamento de apenas 4\%). Esse desempenho qualitativo é similar entre a mesa d'água e as simulações CFD, enquanto no Fluxovento têm-se discrepâncias significativas. Apesar do fluxo também apresentar uma tendência lateralizada, este percorre a porção superior do espaço interno, o que não ocorre nas outras ferramentas e nem nos modelos presentes na literatura especializada (GARCIA; FUENTES, 2005; BITTENCOURT; CÂNDIDO, 2008). Nota-se uma semelhança no canto inferior do espaço, com a estagnação do ar nas três ferramentas analisadas (Figura 11). Ressalta-se que para construções com igual porosidade, as aberturas no centro (caso 1), produzem taxas de ventilação mais altas do que se as aberturas forem localizadas nas extremidades (modelo 2). No entanto, o caso 1 atinge uma menor região do espaço interno comparado com o caso 2. Para Givoni (1976) a configuração do segundo tem melhor condição de ventilação, pois o ar muda de direção, atingindo uma região interna maior. Essas modificações auxiliam na definição do layout em função do uso do edifício.

No caso 3 (Figura 12), tem-se a configuração de uma ventilação unilateral, com a localização de duas aberturas na fachada a barlavento, o que possibilita um fluxo de ar interno mínimo. Esse fenômeno é percebido na mesa d'água pela ausência de espuma, demonstrando um fluxo interno com velocidades bastante reduzidas e nas simulações pela ausência dos vetores e velocidades próximas a o,om/s. No fluxovento essa simulação não conseguiu ser finalizada, constatando-se uma linha no canto inferior o que torna os resultados incompletos e inconsistentes.

Figura 11 - Fluxo de ar no caso 2 para simulação CFD, fluxovento, mesa d'água e percentual de aproveitamento em relação à velocidade externa, respectivamente
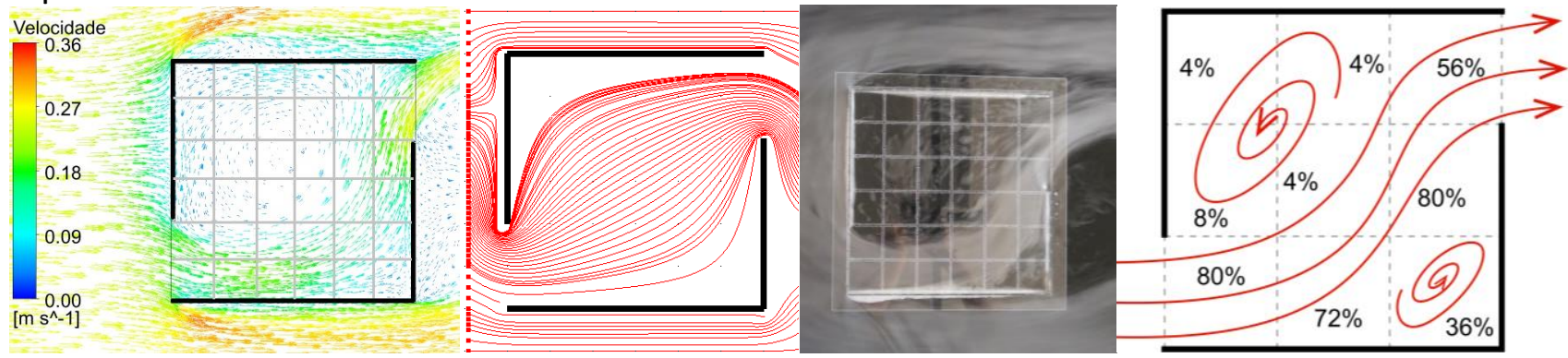

Fonte: os autores. 


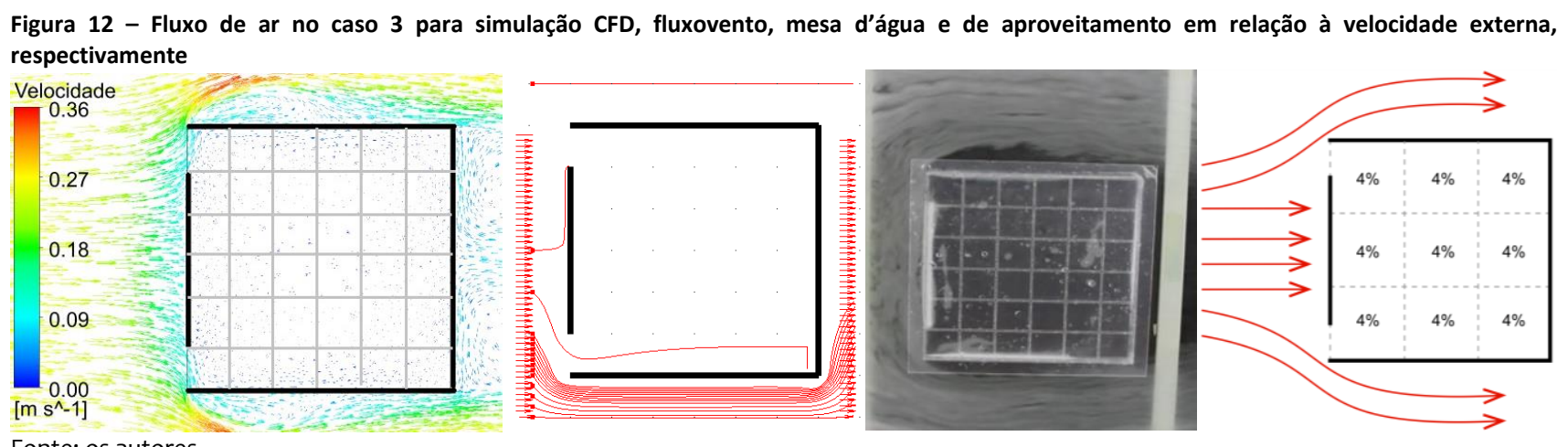

Fonte: os autores.

Nos edifícios de layout livre a ventilação é favorecida, pela permeabilidade dos espaços integrados. Já nos modelos com divisórias internas têm-se maiores recirculações do ar e perdas de energia, devido às mudanças sucessivas de direção do fluxo de ar e pelas expansões e contrações gerada pelas barreiras internas. Dessa forma, têm-se velocidades reduzidas na região posterior desses elementos.

No caso 4, nota-se que o fluxo atravessa o espaço entre as divisórias, cujas dimensões diminuem a região de passagem do ar, com uma aceleração na região posterior, devido ao efeito venturi (aproveitamento de $116 \%$ ). Nas laterais do espaço, são visíveis vórtices representados nos ensaios através da recirculação da espuma e nas simulações pela trajetória dos vetores em círculo. Ressalta-se que as recirculações são mais intensas na mesa d'água em função, principalmente, da viscosidade do fluído, registrando diferenças em relação ao CFD. Na região posterior das divisórias (2 linhas da malha superior e inferior) tem-se uma região sem movimento de ar (aproveitamento de 4\%) (Figura 13), devido a barreira formada pelas divisórias. Assim como nos casos anteriores, o Fluxovento tem significativa discrepância com as simulações CFD e a mesa d'água, pois na região posterior às divisórias tem-se uma distribuição assimétrica com o ar em uma das laterais do ambiente. Ressalta-se que essa ferramenta não possibilita a análise do aumento da velocidade pelo efeito venturi e nem as recirculações do ar, o que omite princípios importantes da ventilação natural.

Para os casos 5 e 6, inseriu-se uma divisória interna modificando a localização das aberturas de entrada e saída de ar. Nesses casos, a divisória fica na frente da abertura de entrada de ar, servindo de bloqueio e desvio do fluxo de ar até a saída, reduzindo a velocidade do ar interno. Com isso, as regiões a sotavento do anteparo possuem velocidades reduzidas com a formação de vórtices e um baixo aproveitamento dos ventos externos (4\%) (Figuras 14 e 15). A configuração do caso 5 mudou o percurso do ar interno, quando comparado ao caso 2. Nesse modelo, a divisória interna permitiu que o fluxo percorresse pelas duas colunas centrais da malha e as duas linhas superiores. A região posterior da divisória não foi atingida pelo fluxo de ar (4\%). No Fluxovento notase a região a sotavento, logo atrás da divisória, sem movimentação de ar, conforme registrado nas outras ferramentas. Porém, na região anterior a divisória, o ar preenche uma maior parte da região superior, o que não foi constatado nas outras ferramentas. 
Figura 13 - Fluxo de ar no caso 4 para simulação CFD, fluxovento, mesa d'água e percentual de aproveitamento em relação à velocidade externa, respectivamente
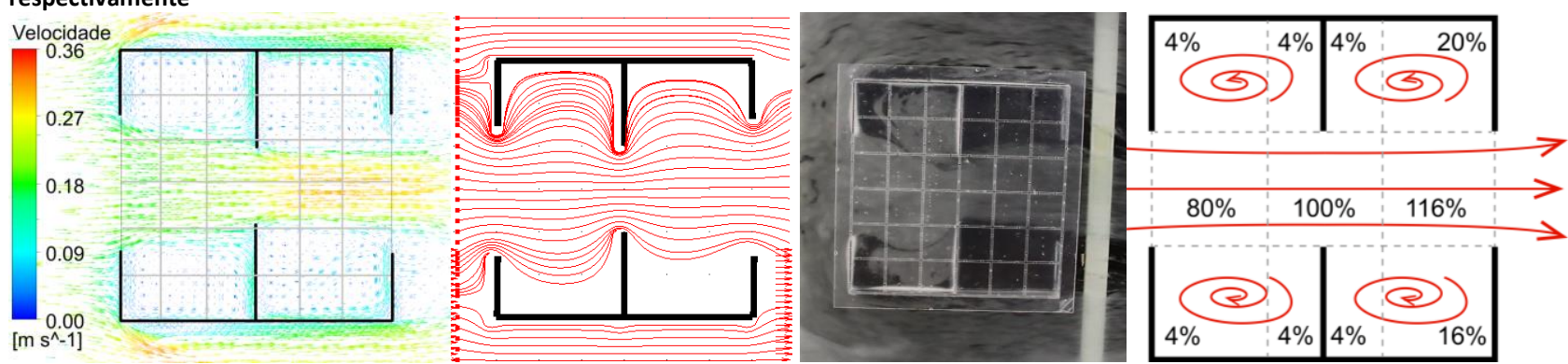

Fonte: os autores.

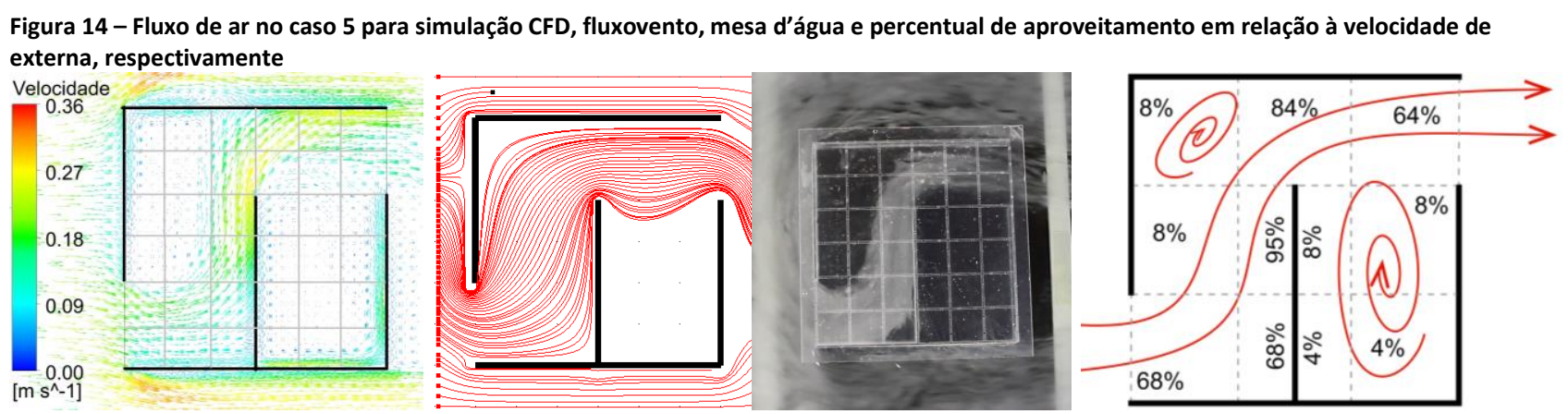

Fonte: os autores.

Figura 15 - Fluxo de ar no caso 6 para simulação CFD, fluxovento, mesa d'água e percentual de aproveitamento em relação à velocidade externa, respectivamente
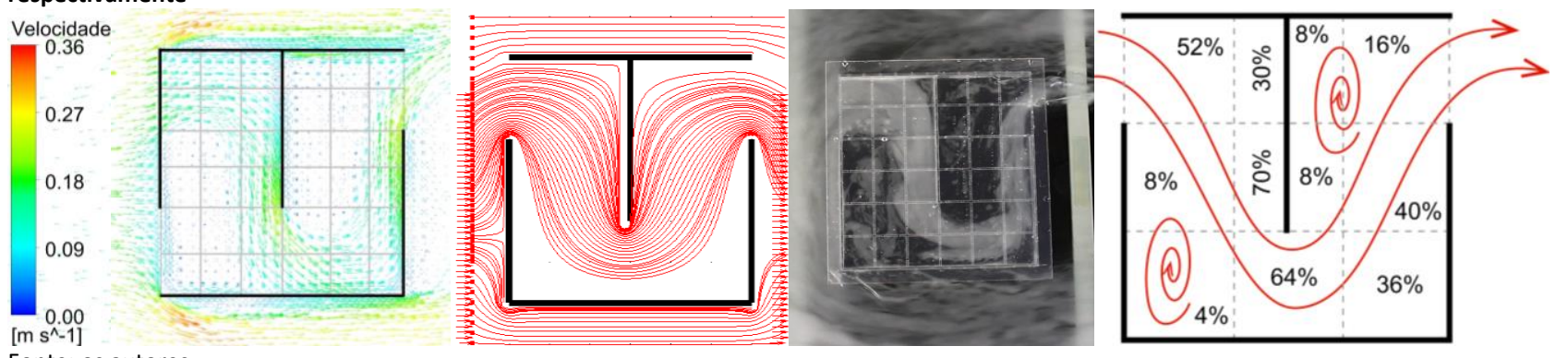

Fonte: os autores.

No caso 6 (Figura 15), se a divisória fosse inexistente, o ar percorreria apenas a região superior do ambiente, no sentido da saída, não atingindo a porção central e inferior do ambiente. Nesse caso, a divisória desviou a trajetória do ar, atingindo uma maior região interna. Nota-se uma significativa semelhança entre as simulações CFD e a mesa d'água, registrando em ambas as ferramentas a curvatura do fluxo de ar na entrada e a proximidade do fluído com a parede a sotavento. No Fluxovento, porém, o ar atinge a região posterior à divisória, o que não acontece no CFD e na mesa d'água, notando-se, novamente, uma diferença significativa da representação qualitativa da ventilação natural. Tanto o software CFD quanto a mesa d'água registram uma região sem movimentação do ar na parte posterior da divisória, o que era esperado, devido à barreira que esse elemento interno ocasiona, o que não foi constatado no Fluxovento.

\section{Conclusão}

Primeiramente, a comparação da aplicabilidade das ferramentas analisadas indica que a mesa d'água e o Fluxovento são mais acessíveis ao ensino para alunos de graduação. No entanto, a análise isolada da aplicabilidade não é suficiente para a definição do seu uso, uma vez que os resultados devem ser confiáveis na representação da ventilação natural. De forma geral, o fenômeno qualitativo da ventilação natural, nos casos analisados, é 
representado pela mesa d'água de forma compatível com as simulações CFD, que é um método de análise de alta confiabilidade. Algumas discrepâncias entre as duas ferramentas foram registradas, como no caso 1. No entanto, para a compreensão dos conceitos básicos da ventilação natural, tais como a escolha da localização das aberturas e da configuração de divisórias internas, a mesa d'água pode auxiliar no ensino com relação aos princípios básicos da ventilação natural e a sua distribuição no espaço interno. Já o software Fluxovento apresentou discrepâncias qualitativas significativas para o entendimento da ventilação natural. Os resultados mostraram diferenças na trajetória do fluxo de ar interno que geram dúvidas sobre a representação do fenômeno com essa ferramenta.

A definição da ventilação natural ocasiona dúvidas nos alunos e até mesmo em projetistas por envolverem análises como a diferença de pressão entre as aberturas, que modificam o desempenho do fluxo de ar interno. Diante disso, essa pesquisa mostra que o uso da mesa d'água, em modelos simplificados, é uma ferramenta confiável para a compreensão desses fenômenos e para o auxílio no ensino e durante a concepção do projeto. Através dos resultados, notou-se que o comportamento do fluído na mesa d'água, se reproduziu dentro da mesma malha nas simulações CFD. Ou seja, para esses modelos simplificados o ensino inicial pode ocorrer por esse equipamento, sem abordar a complexidade do CFD que demandaria um tempo elevado para o aprendizado e para a obtenção dos resultados. No entanto, ressalta-se que em etapas posteriores que exigem definições de parâmetros para um maior detalhamento das estratégias de ventilação natural, como valores de coeficientes de pressão e taxas de renovações de ar/hora é necessário o uso de ferramentas mais complexas como simulações CFD ou ensaios no túnel de vento. O CFD permitiu uma análise mais detalhada e precisa da velocidade do fluxo de ar no ambiente interno, quantificando o aumento ou a redução de velocidade do ar em pontos específicos, úteis para complementar as análises qualitativas. Destaca-se também que as analogias observadas entre a mesa d'água e as simulações por CFD são restritas aos casos simplificados modelados. Situações com outra direção de vento ou diferentes configurações de geometrias mais complexas devem ser previamente avaliadas.

Por fim, outra contribuição do estudo foi à definição e a confecção dos modelos físicos reduzidos com flexibilidade, que pode ser utilizado como base nas disciplinas de conforto ambiental. A escolha por modelos simplificados e existentes na literatura foi visando auxiliar nesse primeiro contato do aluno de graduação com os princípios da ventilação natural. Acredita-se que para o aprendizado dos alunos os modelos devem ser simplificados e que o aprendizado nessa base pode possibilitar, posteriormente, a decisões de projeto mais acertadas.

\section{Referências}

ARAÚJO, C. V. de A. Análise de componentes arquitetônicos para potencialização da ventilação natural com ênfase em captadores de vento. 2011. 130p. Dissertação (Mestrado em conforto no ambiente construído; forma urbana e habitação) - Universidade Federal do Rio Grande do Norte, Programa de Pós-Graduação em Arquitetura e Urbanismo, Natal, 2011.

BITTENCOURT, L.; CÂNDIDO, C. Introdução à Ventilação Natural. 3. ed. Maceió: EdUFAL, 2008. p. 163

BLESSMANN, J. Aerodinâmica das construções. 3. ed. Porto Alegre: Sagra, 2011. p.81.

CALAUTIT, J. K; HUGHES, B. R; CHAUDHRY, H. N; GHANI, S. A. CFD Analysis of a Heat Transfer Device Integrated Wind Tower System for Hot and Dry Climate. Applied Energy, v. 112, p. 576-591, mar. 2013. DOI:

https://doi.org/10.1016/j.apenergy.2013.01.021. 
CALAUTIT, J. K.; HUGHES, B. R. Wind tunnel and CFD study of the natural ventilation performance of a commercial multi-directional wind tower. Building and Environment, v. 80, p. 71-83, oct. 2014. DOI:

https://doi.org/10.1016/j.buildenv.2014.05.022.

CARVALHO, C., MARTHA, L., TEIXEIRA, W. FLUXOVENTO - Um simulador gráfico interativo para o estudo de ventilação em ambientes construídos. In: ENCONTRO NACIONAL DO CONFORTO NO AMBIENTE CONSTRUÍDO, 8., 2005, Maceió. Anais [...] Maceió: ANTAC, 2005.

CÓSTOLA, D. Ventilação Por Ação do Vento no Edifício: procedimentos para quantificação. 2006. 235p. Dissertação (Mestrado em Tecnologia da Construção) - Faculdade de Arquitetura e Urbanismo, Universidade de São Paulo, São Paulo, 2006.

GARRILHO DA GRAÇA, G.; DAISH, N. C.; LINDEN, P. F. A two-zone model for natural cross-ventilation. Building and Environment, v. 89, p. 72-85, jul. 2015. DOI: https://doi.org/10.1016/j.buildenv.2015.02.014.

GARRILHO DA GRAÇA, G.; LINDEN, P. Ten questions about natural ventilation of non-domestic buildings. Building and Environment, v. 107, p. 263-273, oct. 2016. DOI: https://doi.org/10.1016/j.buildenv.2016.08.007.

ETHERIDGE D. W.; SANDBERG, M. Building ventilation: theory \& measurement. 1st edition. Chichester: Jonh Wiles \& sons, 1996. p. 754 .

EVANS, J. M. Housing climate and comfort. London: Architectural Press, 1980.

FRANKE, J.; HIRSCH, C.; JENSEN, A. G.; KRÜS, H. W.; SCHATZMANN, M.; WESTBURY, P. S.; WISSE, J. A.; WRIGHT, N. G. Recommendations on the use of CFD in predicting pedestrian wind environment. In: INT. CONF. ON URBAN WIND ENGINEERING AND BUILDING AERODYNAMICS, 2004. Rhode-Saint-Genèse. Proceedings [... ]. Rhode-SaintGenèse: COST European Cooperation in Science \& Technology. 2004.

FORTUNA, A. O. Técnicas computacionais para dinâmica dos fluidos: conceitos básicos e aplicações. 2. ed. São Paulo: EDUSP, 2000. p 552.

GARCIA , J. R.; FUENTES, V. Viento y arquitectura: el viento como factor de diseño arquitectónico. 3. ed. México: Trillas, 2005. $186 \mathrm{p}$.

GIVONI, B. Man, Climate and Architecture. 2. ed. London: Applied Science Publishers, 1976. p. 499.

GRATIA, E.; BRUYERE, I.; DE HERDE, A. How to use natural ventilation to cool narrow office buildings. Building and environment, v. 39, n. 10, p. 1157-1170, oct. 2004. DOI: https://doi.org/10.1016/j.buildenv.2004.02.005.

HARRIES, A. Notas de aula. In: Workshop: CFX - FAU/USP. São Paulo, 2005.

HUGHES, B. R.; CHAUDHRY H.N.; CALAUTIT, J. K. Passive Energy Recovery from Natural Ventilation air Streams. Applied Energy, v. 40, p. 113- 127, jan. 2014. DOI: https://doi.org/10.1016/j.apenergy.2013.07.019.

KOENIGSBERGER, O.; INGERSOL, T. G.; MAYTHEW, A.; SZOKOLAY, S. V. Manual of tropical Housing and Building. Part I: Climatic Design. Londres: Longman, 1974. p. 336.

KOWALTOWSKI, D. C. C. K.; CELANI, M. G. C.; MOREIRA, D. C.; PINA, S. A. M. G.; RUSCHEL, R. C.; DA SILVA, V. G.; LABAKI, L. C.; PETRECHE, J. R. D. Reflexão sobre metodologias de projeto arquitetônico. Ambiente Construído, v. 6, n. 2, p. 07-19, abr./ jun.2006.

KOWALTOWSKI, D. C. C. K.; LABAKI, L. C.; PINA, S. M. G.; BERTOLLI, S. R. A visualização do conforto ambiental no projeto arquitetônico. In: ENCONTRO NACIONAL DO CONFORTO NO AMBIENTE CONSTRUÍDO, 7., 1998, Florianópolis. Anais [...] Florianópolis: ANTAC, 1998. P. 371-379.

KUKREJA, C. P. Tropical architecture. Nova Dheli: Tata McGraw-Hill, 1978. p.131, 
LAMBERTS, R.; DUTRA, L.; PEREIRA, F. O. R. Eficiência Energética na Arquitetura. 3. ed. São Paulo: PW, 2014. p. 382.

LAWSON, B. How designer think. The design process demystified. 4. ed. London: The architectural Press, 2005. p.320.

LEITE, C. G. Alterações da Ventilação Urbana Frente ao Processo de Verticalização de Avenidas Litorâneas: o caso da avenida litorânea de São Luís/MA. 2008. 229p. Dissertação (Mestrado em Arquitetura e Urbanismo) - Faculdade de Arquitetura e Urbanismo, Universidade de São Paulo, São Paulo, 2008.

MACIEL, A. A. Integração de conceitos bioclimáticos ao projeto arquitetônico. 2006. 197p. Tese (Doutorado em Arquitetura e Urbanismo) - Universidade Federal de Santa Catarina, Florianópolis, 2006.

MATSUMOTO, E; LABAKI, L. Túnel de vento. In: KOWALTOWSKI, D. C. C. K. O processo de projeto em arquitetura: da teoria à tecnologia. São Paulo: Singer, 2011. p. 504.

OLGYAY, V. Design with Climate. New Jersey: Princeton University Press, U.S.A, 1963. p. 224.

PRATA, A. R. Impacto da Altura de Edifícios nas Condições de Ventilação Natural no Meio urbano. 2005. 271p. Tese (Doutorado em Arquitetura e Urbanismo) - Faculdade de Arquitetura e Urbanismo, Universidade de São Paulo, São Paulo, 2005.

PERÉN, J. I.; VAN HOOFF, T.; LEITE, B. C. C.; BLOCKEN, B. CFD analysis of cross-ventilation of a generic isolated building with asymmetric opening positions: impact of roof angle and opening location. Building and Environment, v. 85, p. 263-276, fev. 2015. DOI: https://doi.org/10.1016/j.buildenv.2014.12.007.

ROSSI, M. M.; VALE, F. I.; SHIMOMURA, A. R. P.; CHVATAL, K. M. S. A mesa d'água como ferramenta de apoio para a caracterização de um modelo genérico a ser ensaiado em túnel de vento. Revista IPT: Tecnologia e Inovação, v. 3 , n. 10, p. 70-80, abr. 2019.

TOLEDO, E. Ventilação natural das habitações. 1. ed. Maceió: EdUFAL, 1999. p. 170.

TOLEDO, A.; PEREIRA, F. Potencial da Mesa D’água para a visualização Analógica da Ventilação Natural em Edifícios. In.: ENCONTRO NACIONAL DO CONFORTO NO AMBIENTE CONSTRUÍDO, 7., 2003, Curitiba. Anais [...] Curitiba: ANTAC, 2003. P. 1383-1390.

TRINDADE, S. C.; PEDRINI, A.; DUARTE, R. N. C. Métodos de aplicação da simulação computacional em edifícios naturalmente ventilados no clima quente e úmido. In: Revista do ambiente construído, v.10, n.4, p.37-58, out./dez. 2010. DOI: https://doi.org/10.1590/S1678-86212010000400004.

\footnotetext{
${ }^{1}$ Ana Clara de Almeida Xavier

Estudante de Arquitetura e Urbanismo da Universidade Estadual de Maringá. Endereço postal: Avenida Colombo, 5790, Maringá, PR, Brasil, CEP: 87020-900

2 Izabella Hafele Gularte

Estudante de Arquitetura e Urbanismo da Universidade Federal de Santa Catarina. Departamento de Arquitetura e Urbanismo // CTC-UFSC // Campus Trindade - CP 470 - CEP 88040-970 - Florianópolis (SC).

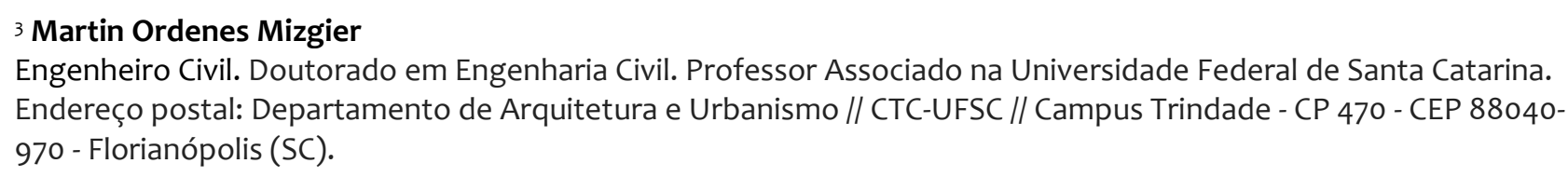

